\title{
Incidence of Speech-Correcting Surgery in Children With Isolated Cleft Palate
}

\section{Gustafsson, Charlotta}

2018-09

Gustafsson, C , Heliovaara , A , Leikola , J \& Rautio , J 2018 , ' Incidence of Speech-Correcting Surgery in Children With Isolated Cleft Palate ', Cleft Palate -

Craniofacial Journal , vol. 55 , no. 8 , pp. 1115-1121 . https://doi.org/10.1177/1055665618760889

http://hdl.handle.net/10138/305917

https://doi.org/10.1177/1055665618760889

unspecified

publishedVersion

Downloaded from Helda, University of Helsinki institutional repository.

This is an electronic reprint of the original article.

This reprint may differ from the original in pagination and typographic detail.

Please cite the original version. 


\title{
Incidence of Speech-Correcting Surgery in Children With Isolated Cleft Palate
}

The Cleft Palate-Craniofacial Journal 2018, Vol. 55(8) ||| $15-|12|$ (c) 2018, American Cleft PalateCraniofacial Association Article reuse guidelines: sagepub.com/journals-permissions DOI: 10.1177/10556656/8760889 journals.sagepub.com/home/cpc (S)SAGE

\author{
Charlotta Gustafsson, MD', Arja Heliövaara, DDS, PhD', \\ Junnu Leikola, MD, DDS, PhD', and Jorma Rautio, MD, PhD'
}

\begin{abstract}
Objective: Speech-correcting surgeries (pharyngoplasty) are performed to correct velopharyngeal insufficiency (VPI). This study aimed to analyze the need for speech-correcting surgery in children with isolated cleft palate (ICP) and to determine differences among cleft extent, gender, and primary technique used. In addition, we assessed the timing and number of secondary procedures performed and the incidence of operated fistulas.

Design: Retrospective medical chart review study from hospital archives and electronic records.

Participants: These comprised the 423 consecutive nonsyndromic children (I57 males and 266 females) with ICP treated at the Cleft Palate and Craniofacial Center of Helsinki University Hospital during 1990 to 2016.

Results: The total incidence of VPI surgery was $33.3 \%$ and the fistula repair rate, $7.8 \%$. Children with cleft of both the hard and soft palate $(n=300)$ had a VPI secondary surgery rate of $37.3 \%$ (fistula repair rate $10.7 \%$ ), whereas children with only cleft of the soft palate $(n=123)$ had a corresponding rate of $23.6 \%$ (fistula repair rate $0.8 \%$ ). Gender and primary palatoplasty technique were not considered significant factors in need for VPI surgery. The majority of VPI surgeries were performed before school age. One fifth of patients receiving speech-correcting surgery had more than one subsequent procedure.

Conclusion: The need for speech-correcting surgery and fistula repair was related to the severity of the cleft. Although the majority of the corrective surgeries were done before the age of 7 years, a considerable number were performed at a later stage, necessitating long-term observation.
\end{abstract}

\section{Keywords}

cleft palate, velopharyngeal insufficiency, pharyngoplasty, fistula

\section{Introduction}

Isolated cleft palate (ICP) is generally divided into clefts of the soft palate (SCP) and clefts of both the soft and hard palate (HSCP). The etiology is thought to be a combination of environmental and genetic factors. ICP is very often associated with other malformations or known genetic syndromes, with reported incidence rates ranging from $21 \%$ to $59 \%$ (Shprintzen et al., 1985; Jones, 1988; Lilius, 1992; Calzolari et al., 2004; Burg et al., 2016).

Generally, ICP is the rarest type of oral cleft (Burg et al., 2016). However, ICP is the prevailing cleft type in Finland, which is known for having a high incidence of oral clefts (Derijcke et al., 1996). The total incidence of oral clefts in Finland is 25.9 per 10000 live births, whereas the corresponding incidence in Europe is 13.4 per 10000 live births. With a rate of 14.7 per 10000 live births, the ICP incidence is significantly higher than elsewhere in Europe, where the rate is on average 5.4 per 10000 live births (National Institute for Health and Welfare, 2017).

The main goal of primary cleft palate surgery is to close the defect and restore the function of the soft palate to allow normal speech and feeding, and to ensure minimal impact on facial growth (Haapanen, 1992; Gart and Gosain, 2014). Previous studies have described velopharyngeal insufficiency (VPI) incidence to range from $5 \%$ to $86 \%$ among children with corrected cleft palate (David et al., 2006; Sullivan et al., 2010;

\footnotetext{
' Cleft Palate and Craniofacial Center, Department of Plastic Surgery, Helsinki University Hospital, Helsinki, Finland

\section{Corresponding Author:}

Charlotta Gustafsson, Cleft Palate and Craniofacial Center, Department of Plastic Surgery, Helsinki University Hospital, P.O. Box 266, FI-00029 HUS, Finland.

Email: charlotta.gustafsson@helsinki.fi
} 
Hosseinabad et al., 2015). The wide variance among studies likely at least partially reflects different methodologies and criteria used in speech assessment.

The cause of VPI is multifactorial and not fully understood. Studies have indicated that the type of cleft and its severity grade, the surgical technique, the surgeon's experience, and the timing of the primary palatoplasty all have an impact on speech outcome (Haapanen, 1992; Andersson et al., 2010; Pearson and Kirschner, 2011; Owusu et al., 2013). VPI is treated by speech therapy and, when necessary, surgery (Andersson et al., 2010; Samoy et al., 2015). One way to assess the success of primary palatoplasty is the need of later VPI surgery or fistula closure (Bicknell et al., 2002).

Many factors may influence the rate of VPI surgery. Severity of the VPI, the patient's and parents' wishes, peer pressure, the speech/language therapist's recommendations, and the surgeon's experience and threshold to perform secondary surgery all play a role. Other factors that might affect surgical interventions are associated anomalies or diseases, additional speech disorders, airway obstruction, and commitment to follow-up visits (Hosseinabad et al., 2015). Children with HSCP have VPI more often than children with SCP (Brunnegård and Lohmander, 2007; Hosseinabad et al., 2015), and therefore tend to be treated more often by secondary velopharyngoplasty than SCP children are (Vedung, 1995; Marrinan et al., 1998; Bicknell et al., 2002; Andersson et al., 2010; Parina et al., 2014).

Several surgical techniques exist for management of VPI, and the ideal technique is under dispute (Collins et al., 2012; Gart and Gosain, 2014). The most common procedures today are the pharyngeal flap, usually superiorly located; various sphincter pharyngoplasties; and repair of the soft palate either by the Furlow double-opposing Z-plasty technique or muscle re-repair (Pearson and Kirschner, 2011; Collins et al., 2012; Gart and Gosain, 2014). Many teams favor a tailored approach, choosing the technique according the velopharyngeal closing pattern as seen by videofluoroscopy and nasopharyngoscopy, perhaps even dynamic MRI. At the Cleft Palate and Craniofacial Center of Helsinki University Hospital, we have used a staged approach by usually choosing the Furlow technique and, if this has not led to a good outcome, following this with a superiorly based pharyngeal flap as a secondary procedure.

Speech-correcting surgery can be performed at any age. Surgery is often done before school age (in Finland, before the age of 7 years) to avoid negative social and psychological consequences of poor speech (Becker et al., 2004; de Buys Roessingh et al., 2008). Although the majority of the secondary velopharyngeal repair surgeries are carried out in childhood, a considerable number are corrected at a later stage.

This study aimed to identify and analyze the frequency of secondary speech-correcting surgeries carried out in children with ICP. We evaluated differences between cleft extent, gender, and primary technique used. In addition, we assessed the timing and total number of secondary speech-correcting procedures and the incidence of operated fistulas.

\section{Methods}

In this retrospective study, data on 686 children with ICP were collected over an 11-year period (patients born between 1990 and 2000). All patients were born in Finland and had undergone primary palatoplasty at the Cleft Palate and Craniofacial Center of Helsinki University Hospital. The study protocol was approved by the Helsinki University Hospital. Principles outlined in the Declaration of Helsinki were followed. Medical charts were accessed through the hospital's archive and database. The following data for each patient were retrieved: date of birth, gender, type of cleft, other anomalies, age at primary repair, surgical technique used, and, if performed, the age at and technique of the secondary pharyngoplasty. In addition, the incidence of operated fistula was assessed.

ICPs were divided into total clefts of the hard and soft palate and clefts of the soft palate alone. Submucous cleft palates were not included in this study. Patients with Pierre Robin sequence $(\mathrm{n}=72)$, other associated syndromes, anomalies of the head and neck region, severe hearing loss, other medical conditions, or cognitive disabilities were excluded. Children with van der Woude syndrome $(n=15)$ were retained in the study. Patients with a follow-up time of less than 8 years or whose controls had moved to another cleft center were excluded.

\section{Surgical Methods}

The technique used in the primary closure of the cleft has varied over time. From 1990 to 1991, the clefts were still usually closed at the age of 12 months by the Veau-Wardill-Kilner (V-W-K) technique, which had been in use in the center since it opened in the 1940s. In this technique, much attention was given to force the flaps of the hard palate in a V-Y retroposition in the hope of achieving more length to the soft palate. In 1992 to 1994 , the standard protocol was changed to the Bardach 2-flap technique, which is similar to the V-W-K technique, but the flaps were returned to the donor areas to avoid areas of secondary healing over bone. The timing of palatal closure was simultaneously changed to 9 months of age, which it has since remained. At the end of 1994, a straight-line closure described by Mendoza et al. (1994) became more common, as it was noted that the more extensive raising of flaps in the Bardach method was often not necessary to achieve tensionless closure of the cleft. If tension was noted at the end of the procedure, lateral relaxing incisions were made corresponding to the von Langenbeck technique. In the latter part of the 1990s, the Bardach technique was used as a default for wider clefts and the straight-line/von Langenbeck technique for narrower clefts at the discretion of the individual surgeon.

Surgical management in the nasopharynx region, also known as secondary pharyngoplasty, was carried out to correct VPI. Procedures used were pharyngeal flaps-Honig, where the inferior surface of the flap was unlined, and Hogan, where it was lined, sphincter pharyngoplasty - according to Jackson and Hynes techniques and Furlow palatal re-repair. The 
number of revisions of pharyngeal flaps, usually by elevating the base of the flap with a V-Y plasty and cutting the flap to relieve obstruction, was also calculated.

Symptomatic fistulas with nasal emissions causing distorted speech and/or leakage of fluid and food through the nose were operated.

\section{Assessment of VPI}

The children were examined and followed up by standard protocols of the cleft team containing plastic surgeons, a maxillofacial surgeon, orthodontists, a phoniatrician, speech and language therapists, and an ear, nose, and throat specialist.

Speech was assessed at 3,6, 8, and 10 years up to the age of 16 years, or later if needed. A small number of the children born early in the study population had a shorter follow-up time of 10 years, because of the practice at that time.

Cleft speech characteristics generally related to VPI consisted of the assessment of hypernasality, nasal air emissions, and difficulties in producing the pressure consonants $/ \mathrm{p}, \mathrm{t}, \mathrm{k} /$. To rate VPI symptoms, a 5-point scale was used as follows: normal velopharyngeal competence (0); insignificant, mild, and occasional VPI (1); mild and consistent VPI (2); moderate and consistent VPI (3); and severe and consistent VPI (4). In insignificant VPI (1), nasality or nasal emissions were detected only temporarily, and the speech sound was nearly normal. In addition, pressure consonants were not affected and no speech correction treatment was required. In mild VPI (2), speech was mildly nasal at all times and pressure consonants were not affected. Moderate VPI (3) indicated that speech featured moderate nasality at all times, and a weakness in pressure consonants was detected. In severe VPI (4), speech was highly nasal, and pressure consonants were either weak or replaced by glottal stops.

The decision for speech operations was based on perceptual and instrumental (nasometer and videofluorography) assessment of an experienced cleft phoniatrician and/or speech therapist. The decisions were made together with the plastic surgeons of the cleft team.

\section{Statistical Analysis}

Data were collected with Microsoft Excel and analyzed with the Statistical Package for Social Sciences (SPSS), version 21.0. Comparisons between the groups were done with Pearson $\chi^{2}$ test and Fisher exact test. Differences were considered significant when $P<.05$.

\section{Results}

Altogether, 263 patients of the initial 686 candidates were excluded, which left 423 patients in the study, 157 males (37.1\%) and 266 females $(62.3 \%)$ (Table 1). The median age at the primary palatoplasty was 9 months (range, 6-17 months). The median follow-up was 14.5 years. The total incidence of secondary pharyngoplasty in the study population was $33.3 \%$ $(\mathrm{n}=141)$, and the overall fistula repair rate was $7.8 \%(\mathrm{n}=33)$.
Table I. Population Characteristics of the Total Study Population.

\begin{tabular}{lccc}
\hline & HSCP, $\mathrm{n}$ & SCP, $\mathrm{n}$ & Total, $\mathrm{n}$ \\
\hline Total population & 489 & 197 & 686 \\
Included $^{\mathrm{a}}$ & 300 & 123 & 423 \\
Male & 108 & 49 & 157 \\
Female & 192 & 74 & 266 \\
Excluded & 189 & 74 & 263 \\
Male & 82 & 29 & 111 \\
Female & 107 & 45 & 152 \\
Syndrome & 109 & 16 & 125 \\
Other medical condition & 24 & 11 & 35 \\
Change of CLP center & 28 & 17 & 45 \\
Follow-up under 8 years & 28 & 30 & 58 \\
\hline
\end{tabular}

Abbreviations: HSCP, cleft of the hard and soft palate; SCP, cleft of the soft palate.

${ }^{\mathrm{a}}$ Gender ratio between cleft types in the total population. $\chi^{2}=0.550, P=.458$, $\mathrm{df}=\mathrm{I}$.

Table 2. Rates of Speech-Correcting Surgeries and Fistula Repair After Primary Palatoplasty.

\begin{tabular}{|c|c|c|c|c|c|}
\hline & \multirow[b]{2}{*}{ Total, n (\%) } & \multicolumn{2}{|c|}{ Pharyngoplasty } & \multicolumn{2}{|c|}{ Fistula Repair } \\
\hline & & n (\%) & $P$ & n (\%) & $P$ \\
\hline \multicolumn{6}{|l|}{ Cleft extent } \\
\hline HSCP & $300(70.9)$ & $112(37.3)$ & .006 & $32(10.7)$ & .001 \\
\hline SCP & $123(29.1)$ & $29(23.6)$ & & I (0.8) & \\
\hline \multicolumn{6}{|l|}{ Gender } \\
\hline Female & $266(62.3)$ & $86(32.3)$ & .569 & $23(8.6)$ & .399 \\
\hline Male & $157(37.1)$ & $55(35.0)$ & & $10(6.4)$ & \\
\hline \multicolumn{6}{|c|}{ Primary palatoplasty technique } \\
\hline Bardach & $163(38.5)$ & $60(36.8)$ & .440 & $12(7.4)$ & .000 \\
\hline Langebeck & $37(8.7)$ & $14(37.8)$ & & $2(5.4)$ & \\
\hline Mendoza & $150(35.5)$ & $43(28.7)$ & & $5(3.3)$ & \\
\hline Veau-Wardill-Kilner & $70(16.5)$ & $24(34.3)$ & & $14(20.0)$ & \\
\hline Furlow $^{\mathrm{a}}$ & $3(0.7)$ & $0(0.0)$ & & $0(0.0)$ & \\
\hline
\end{tabular}

Abbreviations: HSCP, cleft of the hard and soft palate; SCP, cleft of the soft palate.

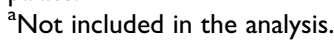

\section{Cleft Extent}

Of the study population, 300 patients $(70.9 \%)$ had an HSCP, whereas 123 patients $(29.1 \%)$ had an SCP. The severity of the cleft was a significant factor in determining the need for secondary pharyngoplasty. In HSCP, the VPI operation rate was $37.3 \%(\mathrm{n}=112)$, whereas the corresponding rate in SCP was $23.6 \%(\mathrm{n}=29)\left(\chi^{2}=7.428, P=.006, \mathrm{df}=1\right)($ Table 2$)$. Also, fistula closure was more often needed in HSCP, $10.7 \%$ $(\mathrm{n}=32)$, than in SCP, $0.8 \%(\mathrm{n}=1)\left(\chi^{2}=11.776, P=.001\right.$, $\mathrm{df}=1)$ (Table 2).

\section{Gender}

The gender ratio between cleft types was not statistically significant. Gender was not associated with VPI surgery or fistula repair rate. Females had a VPI surgery rate of $32.3 \%(\mathrm{n}=86)$ and males $35.0 \%(\mathrm{n}=55)\left(\chi^{2}=0.324, P=.569, \mathrm{df}=1\right)$ 


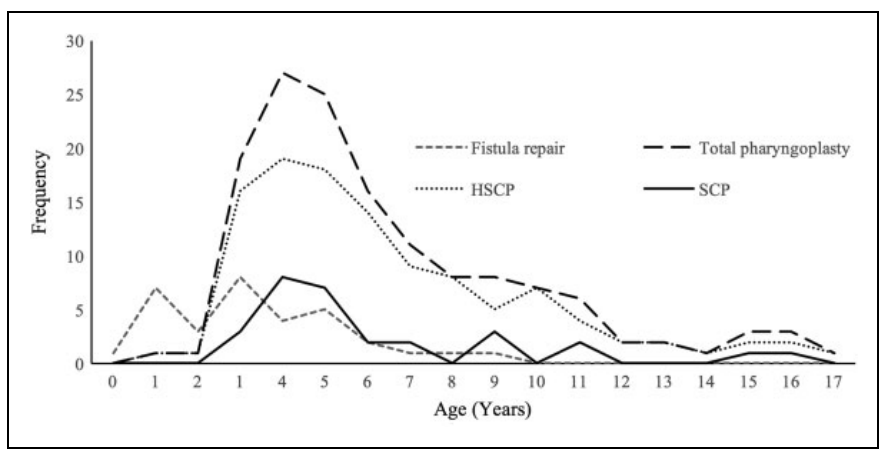

Figure I. Frequency $(n)$ of first pharyngoplasty and fistula repair performed at specific ages (years).

(Table 2). The female fistula closure rate was $8.6 \%(n=23)$, and the corresponding rate for males was $6.4 \%(\mathrm{n}=10)$ $\left(\chi^{2}=0.712, P=.399, \mathrm{df}=1\right)$.

\section{Primary Palatoplasty Technique}

All clefts were closed in a single-step procedure with various techniques. The most frequently used technique was the Bardach 2-flap technique $(\mathrm{n}=163)$, followed by straight-line closure $(\mathrm{n}=150)$. Other techniques used were Veau-WardillKilner $(\mathrm{n}=70)$, von Langenbeck $(\mathrm{n}=37)$, and Furlow $(\mathrm{n}=3)$.

The primary palatoplasty technique, determining the need for speech-correcting surgery, was not a significant variable $\left(\chi^{2}=2.703, P=.440, \mathrm{df}=3\right)$. Meanwhile, a correlation was observed between the primary palatoplasty technique and the fistula repair rate. Fistula closures were more often needed in the Veau-Wardill-Kilner group $(\mathrm{n}=14,20.0 \%)\left(\chi^{2}=18.859\right.$, $P=.000, \mathrm{df}=3$ ). Considering the low frequency of Furlow palatoplasty, this technique was excluded from the analysis (Table 2).

\section{Age and Type of Secondary Pharyngoplasty}

A total of 141 children needed secondary pharyngoplasty $(33.3 \%)$. Of the surgeries, $63.1 \%(\mathrm{n}=89)$ were performed before school age (before 7 years); $61.6 \%(\mathrm{n}=69)$ in HSCP and $68.9 \%(\mathrm{n}=20)$ in SCP. The rest of the surgeries, $36.9 \%$ $(\mathrm{n}=53)$, were done at 7 years of age or later (Figure 1). The mean age at speech-correcting surgery was 5.8 years in the first half of the study population (patients born 1990-1995), whereas the corresponding age in the later population (born 1996-2000) was 7.3 years.

The most frequently used techniques were Furlow pharyngoplasty $(45.4 \%, \mathrm{n}=64)$ and Honig pharyngeal flap technique $(41.8 \%, \mathrm{n}=59)$. The Honig technique was the most common procedure until 2005, when the Furlow repalatoplasty became the standard primary technique in VPI surgery (Table 3).

The majority $(80.1 \%, \mathrm{n}=113)$ of the children requiring pharyngoplasty had only 1 operation, but one-fifth $(19.9 \%$, $\mathrm{n}=28$ ) needed more than 1 procedure. Surgical revisions of pharyngeal flaps were also included. One patient required as many as 5 operations to normalize speech $(P=.828)$ (Table 4$)$.
Table 3. Pharyngoplasty Techniques Performed According to Cleft Extent.

\begin{tabular}{lccccc}
\hline & \multicolumn{5}{c}{ Technique, $n(\%)$} \\
\cline { 2 - 6 } & Honig & Hogan & Jackson & Furlow & Hynes \\
\hline HSCP & $49(43.8)$ & $10(8.9)$ & $2(1.8)$ & $49(43.8)$ & $2(1.8)$ \\
SCP & $10(34.5)$ & $4(13.8)$ & $0(0.0)$ & $15(51.7)$ & $0(0.0)$ \\
Total & $59(41.8)$ & $14(9.9)$ & $2(1.4)$ & $64(45.4)$ & $2(1.4)$ \\
\hline
\end{tabular}

Abbreviations: HSCP, cleft of the hard and soft palate; SCP, cleft of the soft palate.

Table 4. Number of Secondary Surgeries Performed on a Child. ${ }^{\text {a }}$

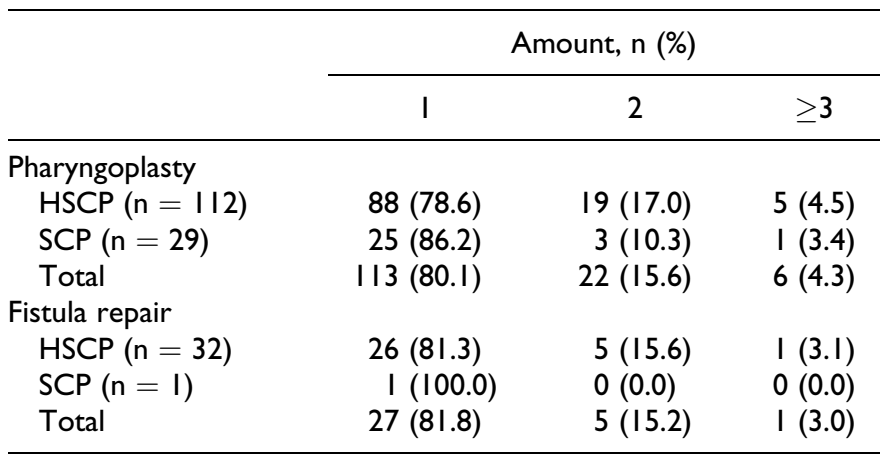

Abbreviations: HSCP, cleft of the hard and soft palate; SCP, cleft of the soft palate.

${ }^{a}$ Total recurrent fistulas: $\mathrm{n}=8(24.2 \%)$.

\section{Fistula Location and Need for Reoperation and Pharyngoplasty}

Fistulas were operated in 33 patients $(7.8 \%)$. One-fourth of these patients $(\mathrm{n}=8,24.2 \%)$ developed a recurrent fistula (Table 5) and almost one-fifth $(\mathrm{n}=6,18.2 \%)$ needed reoperation to close the fistula (Table 4). Twenty-eight (19.9\%) of the children requiring speech-correcting surgery needed fistula repair also either as a single procedure $(\mathrm{n}=10)$ or simultaneously with the pharyngoplasty $(\mathrm{n}=18)$. All operated fistulas, except for one, were found in children with HSCP. No significant correlation was observed between the location of the operated fistulas, the need for reoperation of the fistulas, or the need for pharyngoplasty and fistula closure $\left(\chi^{2}=3.596\right.$, $P=.166, \mathrm{df}=2)(P=.355)(P=1.000)($ Table 5$)$.

\section{Discussion}

Previous studies investigating children with ICP have reported speech-correcting surgery rates of $14 \%$ to $29 \%$ (Heliövaara et al., 1992; Rintala and Haapanen, 1995; Marrinan et al., 1998; Bicknell et al., 2002; Inman et al., 2005; Andersson et al., 2010; Nyberg et al., 2010; Lithovius et al., 2014; Parina et al., 2014). Our study revealed a slightly higher incidence of $33 \%(n=141 / 423)$ in the total population.

It is commonly emphasized that cleft extent correlates with speech outcome. We confirm that children with a less severe cleft had less need for VPI surgery than those with a more 
Table 5. Location of Fistula and Need for Reoperation and Pharyngoplasty.

\begin{tabular}{lccccrrr}
\hline Fistula location & Fistula Closure & $P$ & Recurrent Fistula & $P$ & Pharyngoplasty & $P$ & $\begin{array}{c}\text { Combined Fistula Closure } \\
\text { and Pharyngoplasty }\end{array}$ \\
\hline Hard palate & $18(54.5)$ & .166 & $6(33.3)$ & $.355^{\mathrm{a}}$ & $15(83.3)$ & $1.000^{\mathrm{a}}$ & $9(39.1)$ \\
Junction of hard and soft palate & $6(18.2)$ & & $0(0.0)$ & & $5(83.3)$ & $.636^{\mathrm{a}}$ \\
Soft palate & $9(27.3)$ & & $2(22.2)$ & $8(88.9)$ & $4(44.4)$ & $5(27.8)$ & $18(36.0)$ \\
Total & $33(100.0)$ & $8(24.2)$ & $28(84.8)$ & \\
\hline
\end{tabular}

${ }^{\mathrm{a}}$ Fisher exact test.

severe form. Children with SCP had a $23.6 \%$ rate for speechcorrecting surgery, whereas children with HSCP had a rate of $37.3 \%$. The difference was significant $(P=.006)$. Several studies have shown similar results. Andersson et al. (2010) reported that $\mathrm{HSCP}$ had a $42 \%$ incidence of pharyngoplasties compared with SCP, which had a significantly lower incidence of $10 \%$. Bicknell et al. (2002) described almost identical results in their study, with a $44 \%$ rate in the HSCP group and a $12 \%$ rate in the SCP group. Marrinan et al. (1998), in turn, observed a lower flap incidence of $23 \%$ in the HSCP group, whereas the incidence in the SCP group remained at $10 \%$.

In the literature, several theories explaining speech outcome and relation to cleft severity have been presented. Marrinan et al. (1998) stressed the importance of the vomer and the extent of its attachment to the palatal shelves. In SCP, the vomer is attached to both palatal shelves, whereas in HSCP the vomer is separated from the surrounding dysplastic and disorientated musculature and is combined with shortened shelves (Marrinan et al., 1998; Bicknell et al., 2002). This is reflected in speech outcome.

No important differences emerged between genders of children with ICP and need for speech-correcting surgery. Similar results have been reported in another study encompassing only cleft of the isolated palate (Andersson et al., 2010). Some studies have shown significant variation in speech outcome (Bicknell et al., 2002; Lithovius et al., 2014); the diversity might be explained by the wide spectrum of cleft types in the analyzed populations.

The technique used in the primary palatoplasty was not a significant factor regarding need for secondary VPI surgery. Many studies have examined whether the technique of initial palatoplasty plays a crucial role in subsequent need for corrective surgery. Results obtained are not unambiguous; some show no correlation, while others show a trend for an association (Marrinan et al., 1998; Bicknell et al., 2002; Lithovius et al., 2014). Multiple surgeons performing several different techniques probably affect these results. For instance, Rintala and Haapanen (1995) observed that the incidence of speech-correcting surgery correlates strongly with the surgeon's experience. On the other hand, most of the primary surgery in our study $(90 \%)$ was done by 4 experienced cleft surgeons. After the Veau-Wardill-Kilner method was abandoned, the method for primary surgery was determined individually according to the original extent and anatomy of the cleft.
Another interesting aspect of this study is the number of pharyngoplasties performed on a child. One-fifth (19.9\%) of the ICP children receiving VPI management via surgery needed 2 or more surgeries. The HSCP group had a slightly higher incidence $(21.4 \%)$ for surgery than the SCP group (13.8\%), although the difference was not significant.

Speech-correcting surgery is an effective means of treating VPI, and studies indicate that the surgery can be performed at any age. The optimal surgical technique for VPI remains open, as does the optimal age for speech-correcting surgery. Children with speech difficulties rapidly develop inadequate speech patterns trying to compensate VPI. Thus, it is commonly believed that early management of VPI might prevent these changes and reduce the need for later speech therapy. Speech difficulties often carry devastating consequences for social relationships and psychological health of affected individuals. Therefore, in Finland the aim is to undertake surgery in children with VPI before school age. When examining the secondary pharyngoplasties in our study, we found that the majority $(63.1 \%)$ were performed before school age (in Finland, the average age at start of school is 7 years), thus supporting the results of Andersson et al. (2010). By contrast, Vedung (1995) reported that $70 \%$ of the secondary procedures took place at a slightly older age, 5-9 years.

Some studies propose an unfavorable association between increasing age at VPI management and speech outcome. Despite these theories, very few studies have been done concerning this matter. Becker et al. (2004) compared age at VPI surgery with the amount of speech therapy needed postoperatively. They found no significant differences in the number of postoperative speech therapy sessions between children receiving early surgery and children receiving later surgery. VPI has often been described to fluctuate as a child develops due to growing structures in the nasopharynx region. Earlier studies have therefore addressed the importance of frequent and longterm (to adolescence) follow-up visits to identify children with changing speech at a later stage (Vedung, 1995; Park et al., 2000; Sommerlad, 2003; Andersson et al., 2010). When osteotomies are done to correct poor maxillary growth and occlusion in cleft patients, it is important to also assess the effect of these procedures on velopharyngeal competence pre- and postoperatively. In our study population, one patient required, by the time of data collection, postsurgical pharyngoplasty after maxillary osteotomy. The need for long-term follow-up is also supported by our results, considering that $23.4 \%$ of the pharyngoplasties were carried out at an age of 9 years or older. 
The total fistula repair rate in this study was $7.8 \%(\mathrm{n}=33)$. Interestingly, 28 of these 33 children also required speechcorrecting surgery either as a single procedure $(\mathrm{n}=10)$ or simultaneously with the pharyngoplasty $(n=18)$. The total incidence of fistulas was higher than that in a previous study (2\%) of ICP (Andersson et al. 2008) operated by the Langenbeck and Sommerlad techniques. However, the incidence of operated fistulas in our study was significantly related to the technique of palatal repair. Fistula repair was more often needed after the Veau-Wardill-Kilner (20.0\%) technique than after the Bardach (7.4\%), Langenbeck (5.4\%), or Mendoza (3.3\%) techniques. In the Veau-Wardill-Kilner technique, the $\mathrm{V}-\mathrm{Y}$ procedure is performed so that the whole mucoperiosteal flap and the soft palate are retroposed and the palate is lengthened. This leaves an extensive raw area anteriorly and laterally along the alveolar margin with exposed membranous bone. The incidence of operated fistulas was also related to the severity of the cleft, but not to gender. These findings are in agreement with earlier reports (Heliövaara et al., 1993; Muzaffar et al., 2001; Andersson et al., 2008; Phua and de Chalain, 2008; Landheer et al., 2010; Lu et al., 2010).

The retrospective nature of the documenting of data is a limitation of the study. Because of earlier protocols, the follow-up time of some patients was limited to 8 years, although the median follow-up time was 14.5 years. Children who developed VPI at a later age might have been lost because of the shorter follow-up. In addition, possible neuropsychological disorders could not be evaluated, although children with associated syndromes, anomalies of the head and neck region, severe hearing loss, other medical conditions, or cognitive disabilities were excluded.

\section{Conclusion}

Our study contains a large population of children with ICP and long-term results and incidence of speech-correcting surgery. There are several factors that contribute to the difficulty in comparing our results with earlier findings. Other studies have, for instance, relatively small study populations, additional syndromes and anomalies, short follow-up times, and different approaches in speech evaluations. We were surprised by the high incidence of speech-correcting surgeries (33\%) in the total ICP population and the significant difference in the proportions for HSCP and SCP, $37.3 \%$ vs $23.6 \%$. One-fifth of children with ICP received multiple pharyngoplasties and $7.8 \%$ needed closure of the fistula. Although most of the corrective surgeries were performed before school age, a long follow-up is vital considering changing speech during growth to adulthood. To obtain a comprehensive picture of the total incidence of speech-correcting surgeries in cleft children and the differences in incidences for specific cleft types, further studies of other cleft types are needed.

\section{Declaration of Conflicting Interests}

The author(s) declared no potential conflicts of interest with respect to the research, authorship, and/or publication of this article.

\section{Funding}

The author(s) received no financial support for the research, authorship, and/or publication of this article.

\section{References}

Andersson EM, Sandvik L, Semb G, Åbyholm F. Palatal fistulas after primary repair of clefts of the secondary palate. Scand J Plast Reconstr Surg Hand Surg. 2008;42:296-999.

Andersson EM, Sandvik L, Tørdal IB, Åbyholm F. Pharyngoplasty after primary repair of clefts of the secondary palate. Scand J Plast Reconstr Surg Hand Surg. 2010;44:26-30.

Becker DB, Grames LM, Pilgram T, Kane AA, Marsh JL. The effect of timing of surgery for velopharyngeal dysfunction on speech. J Craniofac Surg. 2004;15:804-809.

Bicknell S, McFadden LR, Curran JB. Frequency of pharyngoplasty after primary repair of cleft palate. J Can Dent Assoc. 2002;68: 688-692.

Brunnegård K, Lohmander A. A cross-sectional study of speech in 10year-old children with cleft palate: results and issues of rater reliability. Cleft Palate Craniofac J. 2007;44:33-44.

Burg ML, Chai Y, Yao CA, Magee W, Figueiredo JC. Epidemiology, etiology, and treatment of isolated cleft palate. Front Physiol. 2016;7:67.

Calzolari E, Bianchi F, Rubini M, Ritvanen A, Neville AJ; EUROCAT Working Group. Epidemiology of cleft palate in Europe: implications for genetic research. Cleft Palate Craniofac J. 2004;41: 244-249.

Collins J, Cheung K, Farrokhyar F, Strumas N. Pharyngeal flap versus sphincter pharyngoplasty for the treatment of velopharyngeal insufficiency: a meta-analysis. J Plast Reconstr Aesthet Surg. 2012;65:864-868.

David DJ, Anderson PJ, Schnitt DE, Nugent MA, Sells R. From birth to maturity: a group of patients who have completed their protocol management. Part II. Isolated cleft palate. Plast Reconstr Surg. 2006;117:515-526.

de Buys Roessingh AS, Herzog G, Cherpillod J, Trichet-Zbinden C, Hohlfeld J. Speech prognosis and need of pharyngeal flap for non syndromic vs syndromic Pierre Robin sequence. J Pediatr Surg. 2008;43:668-674.

Derijcke A, Eerens A, Carels C. The incidence of oral clefts: a review. Br J Oral Maxillofac Surg. 1996;34:488-494.

Gart MS, Gosain AK. Surgical management of velopharyngeal insufficiency. Clin Plast Surg. 2014;41:253-270.

Haapanen ML.Factors affecting speech in patients with isolated cleft palate. A methodic, clinical and instrumental study. Scand J Plast Reconstr Surg Hand Surg Suppl. 1992;26:1-61.

Heliövaara A, Rintala A, Ranta R. One-stage closure of isolated cleft palate with the Veau-Wardill-Kilner V to Y pushback procedure or the Cronin modification. I. Comparison of operative results. Scand J Plast Reconstr Surg Hand Surg. 1993;27:49-54.

Hosseinabad HH, Derakhshandeh F, Mostaajeran F, Abdali H, Davari HA, Hassanzadeh A, Kummer AW. Incidence of velopharyngeal insufficiency and oronasal fistulae after cleft palate repair: a retrospective study of children referred to Isfahan Cleft Care Team 
between 2005 and 2009. Int J Pediatr Otorhinolaryngol. 2015;79: 1722-1726.

Inman DS, Thomas P, Hodgkinson PD, Reid CA. Oro-nasal fistula development and velopharyngeal insufficiency following primary cleft palate surgery - an audit of 148 children born between 1985 and 1997. Br J Plast Surg. 2005;58:1051-1054.

Jones MC. Etiology of facial clefts: prospective evaluation of 428 patients. Cleft Palate J. 1988;25:16-20.

Landheer JA, Breugem CC, van der Molen AB. Fistula incidence and predictors of fistula occurrence after cleft palate repair: two-stage closure versus one-stage closure. Cleft Palate Craniofac J. 2010; 47:623-630.

Lilius GP. Clefts with associated anomalies and syndromes in Finland. Scand J Plast Reconstr Surg Hand Surg. 1992;26:185-196.

Lithovius RH, Ylikontiola LP, Sándor GK. Frequency of pharyngoplasty after primary repair of cleft palate in northern Finland. Oral Surg Oral Med Oral Pathol Oral Radiol. 2014;117:430-434.

Lu Y, Shi B, Zheng Q, Hu Q, Wang Z. Incidence of palatal fistula after palatoplasty with levator veli palatini retropositioning according to Sommerlad. Br J Oral Maxillofac Surg. 2010;48:637-640.

Marrinan EM, LaBrie RA, Mulliken JB. Velopharyngeal function in nonsyndromic cleft palate: relevance of surgical technique, age at repair, and cleft type. Cleft Palate Craniofac J. 1998;35:95-100.

Mendoza M, Molina F, Azzolini C, Ysunza Rivera A. Minimal incision palatopharyngoplasty: a preliminary report. Scand J Plast Reconstr Surg Hand Surg. 1994;28:199-205.

Muzaffar AR, Byrd HS, Rohrich RJ, Johns DF, LeBlanc D, Beran SJ, Anderson C, Papaioannou A. Incidence of cleft palate fistula: an institutional experience with two-stage palatal repair. Plast Reconstr Surg. 2001;108:1515-1518.

National Institute for Health and Welfare (THL). Congenital anomalies 1993-2011, statistical report, Helsinki: 2017. Available at: www.thl.fi. Accessed September 1, 2017.

Nyberg J, Westberg LR, Neovius E, Larson O., Henningsson G. Speech results after one-stage palatoplasty with or without muscle reconstruction for isolated cleft palate. Cleft Palate Craniofac J. 2010;47:92-103.

Owusu JA, Liu M, Sidman JD, Scott AR. Does the type of cleft palate contribute to the need for secondary surgery? A national perspective. Laryngoscope. 2013;123:2387-2391.

Parina RP, Chang DC, Saad AN, Tokin CA, Gosman AA. Statewide multicenter analysis of the incidence of secondary surgeries after isolated cleft palate repair. Ann Plast Surg. 2014;72:71-75.

Park S, Saso Y, Ito O, Tokioka K, Takato T, Kato K, Kitano I. The outcome of long-term follow-up after palatoplasty. Plast Reconstr Surg. 2000;105:12-17.

Pearson GD, Kirschner RE. Surgery for cleft palate and velopharyngeal dysfunction. Semin Speech Lang. 2011;32:179-190.

Phua YS, de Chalain T. Incidence of oronasal fistulae and velopharyngeal insufficiency after cleft palate repair: an audit of 211 children born between 1990 and 2004. Cleft Palate Craniofac J. 2008; 45:172-178.

Rintala AE, Haapanen ML. The correlation between training and skill of the surgeon and reoperation rate for persistent cleft palate speech. Br J Oral Maxillofac Surg. 1995;33:295-298.

Samoy K, Hens G, Verdonck A, Schoenaers J, Dormaar T, Breuls M, Vander Poorten V. Surgery for velopharyngeal insufficiency: the outcomes of the University Hospitals Leuven. Int J Pediatr Otorhinolaryngol. 2015;79:2213-2220.

Shprintzen RJ, Siegel-Sadewitz VL, Amato J, Goldberg RB. Anomalies associated with cleft lip, cleft palate, or both. Am J Med Genet. 1985;20:585-595.

Sommerlad BC. A technique for cleft palate repair. Plast Reconstr Surg. 2003;112:1542-1548.

Sullivan SR, Marrinan EM, Mulliken JB. Pharyngeal flap outcomes in nonsyndromic children with repaired cleft palate and velopharyngeal insufficiency. Plast Reconstr Surg. 2010;125:290-298.

Vedung S. Pharyngeal flaps after one- and two-stage repair of the cleft palate: a 25-year review of 520 patients. Cleft Palate Craniofac J. 1995;32:206-215. 